\title{
Estrategias didácticas para la enseñanza del inglés en educación media general
}

\section{Didactic Strategies for English Teaching in General Secondary Education}

Keila Esther Guerrero Chacón. ${ }^{1}$, Carina Fernanda Vallejo Barreno. ${ }^{2}$ \& María Soledad Caicedo

Valencia. $^{3}$

Recibido: 10-09-2019 / Revisado: 03-10-209 /Aceptado: 04-11-2019/ Publicado: 05-12-2019

\begin{abstract}
DOI: https://doi.org/10.33262/cienciadigital.v3i4.2.1001

The objective of the research was to propose teaching strategies for teaching English in General Secondary Education in the San Francisco Municipality, Zulia state; framed in the educational supervision research field. The research is descriptive, under the modality of feasible field project; with a non-experimental and cross design. To do so, a population of 11 managers, 59 teachers from different subjects and 6 English teachers was selected. A survey as an instrument for data collection was developed, being this dichotomous under the KuderRichardson scale, composed by 30 items and validated by 5 fourth-level expert academic peers. It was applied to a sample of 20 subjects with the same characteristics to the study population but from different institutions, obtaining high reliability with the value of 0.892 . The research has met with systematic phases where its current situation and antecedent has been observed in order to design $\mathrm{n}$ instrument of data collection. As a result, it was obtained that the surveyed population is identified with the study variable, although the students present weaknesses in the formation of social and self-control skills, as well as in the use of post-instructional strategies and in the development of communicative competence; that is, written comprehension, written and oral expression.
\end{abstract}

Keywords: Teaching strategies, communicative competence, learning, comprehension, expression.

\section{Resumen}

El objetivo de la investigación fue proponer estrategias didácticas de enseñanza del inglés en Educación Media General del Municipio Escolar San Francisco 1, del estado Zulia; enmarcado en la línea de investigación de supervisión educativa. La investigación es

\footnotetext{
${ }^{1}$ Escuela Superior Politécnica de Chimborazo, Centro de Idiomas, Riobamba, Ecuador., keila.guerrero@espoch.edu.ec

${ }^{2}$ Escuela Superior Politécnica de Chimborazo, Facultad de Administración de Empresas, Riobamba, Ecuador, carina.vallejo@espoch.edu.ec

${ }^{3}$ Pontificia Universidad Católica del Ecuador, Facultad de Comunicación, Lingüística y Literatura, Quito, Ecuador, mscaicedo@puce.edu.ec
} 
descriptiva, bajo la modalidad de proyecto factible, de campo; con su diseño no experimental; transversal. Para ello, se seleccionó una población de 11 directivos, 59 docentes de diferentes áreas y 6 docentes de inglés. Se elaboró un instrumento tipo encuesta, siendo este dicotómico bajo la escala de Kuder-Richardson, compuesto por 30 ítems y validado por 5 pares académicos expertos de cuarto nivel. Se aplicó a una muestra de 20 sujetos con iguales características a la población de estudio, pero de diferentes instituciones, obteniendo una alta confiabilidad con el valor de 0,892. La investigación cumplió con fases sistemáticas donde se observó su situación inicial y antecedente con el fin de diseñar un instrumento de recolección de datos. Como resultado se obtuvo que la población encuestada se identifica con la variable de estudio, aunque los estudiantes presentan debilidades en la formación de las habilidades sociales y de autocontrol, así como en el uso de las estrategias pos instruccionales y en el desarrollo de la competencia comunicativa; es decir la comprensión escrita, expresión escrita y oral.

Palabras claves: Estrategias de enseñanza, competencia comunicativa, aprendizaje, comprensión, expresión

\section{Introducción}

En la actualidad se están viviendo cambios acelerados que involucran todos los aspectos de la vida y en especial el que hacer educativo ya que, el desarrollo del conocimiento se ha venido multiplicando de año en año con más fuerza de acuerdo al avance de la ciencia y la tecnología. Las necesidades exigen un cambio y una evolución que vaya a la par de los últimos acontecimientos.

Gómez (2005) afirma que, "De los cuarenta millones de usuarios estimados de la Internet, la mayoría se comunican en inglés. El 80\% de la información del mundo guardada electrónicamente está en inglés". (p.2). Por tanto, aprender esta lengua es, sin duda, no sólo un medio de acceso a la información y comunicación sino también para la cooperación e intercambio y acercamiento a otras culturas o sistemas de vida.

En tal sentido, se confirma la importancia del dominio de este idioma como segunda lengua. Sin embargo, existe la percepción de que los estudiantes de educación media, tienen poco interés en adquirir los conocimientos que se les ofrece en el área de inglés, pues sus esfuerzos se dirigen solo a superar las evaluaciones que les permitirán aprobar la asignatura, es decir lo conciben con carácter obligatorio para obtener el título de Bachiller.

Alcedo (2002), Chacón (2003), Guerra (2004) hacen un breve recuento de los métodos que se han utilizado desde hace unas cuatro décadas en los procesos de enseñanza y aprendizaje del inglés como Lengua extranjera en el contexto venezolano. Al respecto, mencionan que hasta la década de los 80 hubo un marcado predominio del conductismo y estructuralismo como teorías psicolingüísticas para fundamentar la metodología de la enseñanza del inglés, con énfasis en el conocimiento de una gramática normativa y descriptiva. Esta tendencia fue parcialmente 
sustituida a finales de los 80 , por el advenimiento del enfoque comunicativo instaurado desde entonces en los programas de educación media venezolanos.

Aunque el enfoque comunicativo está centrado en la comunicación e interacción social mediante la lengua extranjera, la práctica educativa muestra, que en su mayoría, los docentes especialistas tienden a enseñar el idioma de manera tradicional, utilizando generalmente un libro texto como único recurso didáctico y con predominio del método de gramática traducción, con repetición mecánica de fórmulas y estructuras gramaticales, que al ser presentadas de manera automatizada al alumno, terminan por desmotivarlo y aislarlo del sentido funcional del lenguaje.

Otro aspecto es el enfrascamiento hacia la enseñanza de una sola o algunas destrezas comunicativas, sabiendo que en la enseñanza de los idiomas se desarrolla la competencia comunicativa hacia las cuatro destrezas que son: comprensión oral, comprensión escrita, expresión oral y expresión escrita. De tal manera que, si se hace un balance entre estas cuatro destrezas, el estudiante pudiese encontrar más variadas y amenas sus clases y le permitiría desarrollar sus potenciales al máximo en varias o en todas estas.

González (2008) desarrolla en la Universidad Pedagógica Nacional Unidad 26 “A” Hermosillo, para optar al grado de Magister en Educación, una investigación denominada "Comunicación y educación en la enseñanza del inglés" El uso de los materiales auténticos como apoyo didáctico en el proceso de enseñanza-aprendizaje del idioma inglés. En este estudio se elaboró un diagnóstico sobre la percepción que tienen los profesores de inglés acerca del uso de los materiales auténticos como apoyo didáctico en el proceso de enseñanza- aprendizaje en la clase de inglés de primera etapa de educación secundaria.

Donde los estudiantes indican que la mayoría de las veces, el profesor de inglés expone en clase el propósito del tema a aprender y que éste domina en su mayoría los objetivos. Por otra parte, los profesores manifiestan que existe un uso regular del material de apoyo en clase, pero que casi nunca éste es material autentico.

Ferrer (2008) en la investigación denominada "Estrategias de comprensión lectora para estudiantes de Educación Media, Diversificada y Profesional en el área de inglés”, evidenció limitaciones para alcanzar la comprensión lectora de textos en inglés y la poca utilización de estrategias cognitivas y metacognitivas por parte de los docentes. A partir del diagnóstico y de la revisión bibliográfica se diseñaron algunas estrategias con el fin de ofrecer alternativas a los docentes para mejorar la comprensión.

Boix (1999) sostiene que "la palabra estrategia aplicada al ámbito didáctico, se refiere a aquella secuencia ordenada y sistematizada de actividades y recursos que los profesores utilizamos en nuestra práctica educativa; determina un modo de actuar propio y tiene como principal objetivo facilitar el aprendizaje de nuestros alumnos" (p.55). De tal manera que, se puede definir a la estrategia, como la actividad que se lleva a cabo de manera premeditada, sistemática y ordenada 
utilizando una gran variedad de recursos didácticos para lograr la comprensión de los contenidos por parte de los estudiantes.

Por otra parte, Orellana (2008) establece que las estrategias tienen como finalidad, que el alumno sea capaz de plantearse objetivos y metas, que permiten al profesor identificar que el estudiante, sabe de qué trata el tema u objetivo; así también la finalidad de su instrucción. Por tanto, las estrategias de inicio son de gran importancia ya que marcan el camino del aprendizaje en cada clase.

Según Alfonzo (2003) la estrategia coinstruccional "es el momento donde se presenta y se procesa la nueva información, dando la oportunidad de procesarla y practicarla hasta manejarla con facilidad" (p.10). Por lo que es necesario preparar las minuciosamente las estrategias, pues se ubican dentro del desarrollo de la clase y es donde los aprendizajes serán internalizados por los estudiantes.

Luengo (2011) afirma que: "Las estrategias pos instruccionales son aquellas formas técnicas metodologías que implican o contienen elementos de evaluación afectiva y cognitiva; en su mayoría están centradas en la visión de la globalización y del logro de los aprendizajes permanentes; lo que implica, que es un tanto difícil establecer actividades concretas ya que el conocimiento solo puede ser aprendido en términos de interacción con el entorno, a través de una metodología interactiva". (p. 58). Es decir que, las estrategias no solo propician una visión global de los aprendizajes, sino también cumplen un papel evaluador del aprendizaje. Por lo que es necesario que se ajusten flexiblemente a las técnicas y contenidos utilizados, procurando lograr una interacción constante entre el grupo y el docente mediador de los aprendizajes.

Pérez (2007) sostiene que la competencia lingüística comunicativa, se refiere a la utilización del lenguaje tanto de la lengua propia como de la(s) extranjera(s) fundamentalmente como instrumento de comunicación oral y escrita, de aprendizaje, de construcción del propio pensamiento y también como instrumento de regulación personal y de regulación de la propia conducta. De tal manera que va involucrada al comportamiento y la conducta como medio para expresar un mensaje y ser entendido por los demás.

Según Baralo (2000) la expresión oral constituye una destreza o habilidad de comunicación que no tiene sentido sin la comprensión, sin el procesamiento y la interpretación de lo escuchado La expresión oral implica la interacción y la bidireccionalidad en un contexto compartido, y en una situación en la que se deben negociar los significados (p.164). Es decir, que es importante estudiar la destreza de la expresión oral en conjunto con las demás para lograr una comunicación efectiva

Zamora (2009) plantea que "la expresión escrita consiste en sacar afuera los pensamientos, las ideas y los sentimientos y plasmarlos en un papel" (p.1). Es decir, que es la acción de plasmar o exponer de manera y ordenada los pensamientos o ideas que tenga a bien el individuo expresar. Partiendo de la habilidad de la expresión, se puede afirmar que la destreza de la expresión escrita 
es fundamental desarrollarla para que el ser humano se pueda comunicar y expresar lo que quiere, de manera escrita.

Carbonel (2008) plantea que "la comprensión oral responde a mecanismos cerebrales comprometidos con determinadas estructuras organizativas lógicas que conducen a traducir inteligentemente lo que se nos dice" (p.223). Por tanto, la comprensión oral lleva a ciertos y serias conexiones y procesamientos neurolingüísticos que hacen la codificación del mensaje emitido para así producir una respuesta coherente al emisor.

Pérez (2007) establece que la comprensión escrita "comprende la habilidad que permite, a partir de distintos tipos de textos, buscar, recopilar y procesar información y ser competente a la hora de comprender" (p.65). Así que, la comprensión escrita no significa solo hacer conexiones de palabras de manera correcta sino de poderlas procesar mentalmente para lograr comprender lo que el autor quiere expresar.

\section{Metodología}

Se asumió la población de (11) directivos, (06) docentes de inglés y (59) docentes de otras áreas, todos pertenecientes a las instituciones: U. E.P. San José de Calasanz, la E.B. Gral Rafael Urdaneta y la E.B. Marie Poussepin, Los cuales están representados en el siguiente cuadro.

La población de la investigación está representada por 03 (tres) instituciones de Educación Media General, pertenecientes al Municipio Escolar San Francisco 1, entre las cuales tenemos a la U.E.P. San José de Calasanz, la E.B. Gral. Rafael Urdaneta y la E.B. Marie Poussepin.

Se consideró un plan o estrategia para alcanzar los objetivos planteados en el estudio. Para el presente caso de tipo no experimental. Así mismo, la investigación es transversal o transeccional, dado que los datos fueron recolectados en un solo momento durante un tiempo único.

El estudio fue de campo, ya que se realizó un análisis sistemático de problemas con el propósito de detallarlos, explicar sus causas y efectos, entender su naturaleza y factores contribuyentes a predecir su ocurrencia, en el medio donde se suscitan los eventos investigados.

En tal sentido, la investigación se enmarcó dentro de la objetividad y no obstante no son dirimentes, las creencias o inclinaciones personales de los evaluadores o de quien, en este caso lleva la autoría del estudio. Así también, se considera total imparcialidad al momento de la seleccionar la población, muestra, evaluación y análisis de los resultados.

Uno de los principios es que la evaluación es independiente de las inclinaciones personales, valores, creencias y nociones idiosincráticas de los evaluadores, quienes tratan de mantenerse alejados de la influencia que puede darse en la obtención de datos, ser imparciales, en lo posible, en el proceso de la evaluación e inclinarse para estudiar poblaciones grandes, que, para fines de inferencias, usan muestras representadas y aleatorias (p.40). 
La investigación es descriptiva ya que, expresó los hechos con sus características tal y como se presentaron para luego analizar los hechos. En tal sentido, Hernández et al. (2006) plantean que "la investigación descriptiva busca especificar propiedades, características y rasgos importantes de cualquier fenómeno que se analice" (p.119). Así mismo, es una investigación cuantitativa bajo enfoque positivista. Al respecto, Barragán et al (2003) refieren que "lo cuantitativo es perteneciente o relativo a la cantidad y de su análisis se determina las porciones de cada elemento analizado" (p.115). De tal manera que se ha totalizando la cantidad de datos, mismos que fueron analizados para llegar a las conclusiones finales. En cuanto a lo positivista; de acuerdo a lo planteado por Quispe, A. (2004):

El estudio se llevó a cabo bajo la modalidad de proyecto factible tipo propuesta, apoyado en una investigación descriptiva y documental, que trató de solucionar problemas, requerimientos y necesidades de un grupo social específico, formulando y llevando a cabo, una serie de programas, métodos y procesos.

Tabla 1. Población

\begin{tabular}{lrrrr}
\hline \multicolumn{1}{c}{ INSTITUCIONES } & DIRECTIVOS & $\begin{array}{c}\text { DOCENTES DE } \\
\text { ENGLATIVAS }\end{array}$ & $\begin{array}{c}\text { DOCENTES DE } \\
\text { OTRAS AREAS }\end{array}$ & \multicolumn{2}{c}{ TOTAL } \\
\hline E.B. Gral. Rafael Urdaneta & 6 & 3 & 34 & 43 \\
U.E.P. San José de Calasanz & 3 & 2 & 15 & 20 \\
E.B. Marie Poussepin & 2 & 1 & 10 & 13 \\
TOTAL & 11 & 6 & 59 & 76 \\
\hline
\end{tabular}

Fuente: Municipio Escolar San Francisco 1

Elaborado por: Guerreo Keila, 2013

La muestra representa la totalidad de la población estratificada a través del censo poblacional, dado que la cantidad de sujetos es bastante accesible y suficiente para recoger los datos esenciales que permitan sondear todos los componentes involucrados en el estudio, y de esta manera minimizar las oportunidades de exclusión de elementos importantes que puedan enriquecer la investigación

Para efectos de la investigación, la variable estrategias didácticas para la enseñanza del inglés en Educación Media General, fue medida a través de las dimensiones: Factores relacionados con el desempeño académico, con los indicadores: Motivación escolar, Autocontrol y habilidades sociales; la dimensión Tipos de estrategias, cuyos indicadores son: preinstruccionales, coinstruccionales y posinstruccionales; y la dimensión Competencia comunicativa, la cual tiene como indicadores: Expresión oral, Expresión escrita, Comprensión oral y Comprensión escrita.

Se utilizó para recolección de datos, una encuesta que permitió recolectar la información directamente de la realidad en la que se encuentran las instituciones educativas a investigar. Para el desarrollo de la encuesta se diseñó un cuestionario dirigido en tres versiones, al personal 
directivo, a los docentes de todas las áreas y a los docentes de inglés, pertenecientes a las tres instituciones a investigar, adscritas al Municipio Escolar San Francisco 1.

Para este estudio se utilizó como instrumento, el cuestionario el cual permitió recoger la información o los datos requeridos, pertinentes y esenciales para la prosecución del estudio directamente de los directivos y docentes. El cuestionario fue de tipo dicotómico de respuesta cerrada, "si" y "no"; compuesta por 30 ítems, elaborado por el autor del trabajo de investigación; este contempla los objetivos específicos, la variable de estudio, las dimensiones e indicadores, considerados en la investigación.

Para la validación del instrumento se solicitó la opinión de 5 expertos, quienes analizaron la pertinencia de los ítems con los indicadores, dimensiones y objetivos, así como la redacción de los mismos. El aporte de cada uno de estos expertos permitió la elaboración del instrumento definitivo.

Para determinar la confiabilidad del instrumento, se aplicó una prueba piloto en 20 sujetos (15 docentes y 5 directivos) que no pertenecen a la población. La prueba piloto fue aplicada en la E.T.I. Cap. Anselmo Belloso y posteriormente se procesaron los datos con la formula Alfa Cronbach, la cual es:

$$
r_{\pi}=\frac{K}{K-1}\left[1-\frac{\sum S_{i}^{2}}{S_{t}{ }^{2}}\right]
$$

Donde:

Aplicando la fórmula de Alfa Cronbach, se obtuvo que el coeficiente de confiabilidad de $\mathrm{rtt}=$ 0,8921 . Lo cual indica que el instrumento es altamente confiable para ser utilizado como instrumento de recolección de datos y aplicado a la población de estudio en esta investigación.

Posterior a la aplicación de los instrumentos de recolección de datos, se procedió con el análisis cuantitativo, iniciando con la tabulación de la información, permitiendo organizar los datos recolectados para luego hacer los cálculos porcentuales con mayor precisión.

Una vez ordenada toda la información, se procedió a su codificación, presentándose en tablas por cada dimensión. En cuanto al paquete estadístico, se utilizó el programa SPSS versión 10.0 para realizar la estadística descriptiva, utilizando el análisis de la descripción de frecuencia absoluta y porcentual de los datos, donde se expone la opinión emitida por parte de los directivos, docentes de inglés y docentes de otras áreas; posteriormente, los resultados fueron confrontados con la teoría respondiendo así a los objetivos planteados al inicio de la investigación para más tarde dar las conclusiones pertinentes.

Para la realización de la investigación se cumplieron las siguientes fases o etapas: 
1. Observación de una situación problema en algunas instituciones de Educación Media General y aprobación de la misma ante el comité académico respectivo.

2. Búsqueda de antecedentes de la investigación

3. Estructuración del planteamiento del problema, objetivos, justificación y marco teórico.

4. Obtención de las listas del personal de las instituciones pertenecientes al Municipio Escolar San Francisco 1, notificándoles el propósito del estudio para contar con la autorización respectiva.

5. Construcción del instrumento de recolección de datos.

6. Validación del contenido del cuestionario por parte de los expertos en el área.

7. Aplicación de la prueba piloto a otros sujetos con características similares a la población de estudio

8. Estimación de la confiabilidad del instrumento a través del método Alfa Cronbach.

9. Aplicación de los instrumentos mediante la realización de visitas programadas a las instituciones educativas, objeto de estudio.

10. Análisis de datos y tabulación.

11. Presentación y discusión de los resultados

12. Conclusiones y elaboración de propuesta.

\section{Resultados}

En la tabla se identifican los factores relacionados al desempeño académico de los estudiantes en el área de inglés del municipio escolar San Francisco 1, del estado Zulia.

Dimensión: Factores relacionados al desempeño académico.

Tabla 2. Identificación de factores de desempeño académico

\begin{tabular}{|c|c|c|c|c|c|c|}
\hline Alternativas & SI & & & NO & & \\
\hline Población & Directivo & Docente & Docente Ingles & Directivo & Docente & Docente Ingles \\
\hline Indicadores & $\%$ & $\%$ & $\%$ & $\%$ & $\%$ & $\%$ \\
\hline Motivación & 93,93 & 89,83 & 94,43 & 6,07 & 10,17 & 5,57 \\
\hline Autocontrol & 90,9 & 81,93 & 88,87 & 9,1 & 18,07 & 11,13 \\
\hline Habilidad Social & 93,93 & 79,67 & 83,33 & 6,07 & 20,33 & 16,67 \\
\hline Promedio & 92,92 & 83,81 & 88,88 & 7,08 & 16,19 & 11,12 \\
\hline Porcentaje & & & $88,54 \%$ & & & $11,46 \%$ \\
\hline
\end{tabular}

Fuente: Cuestionario

Elaborado por: Guerrero Keila, 2013

Al observar la tabla, se puede determinar que el 88,54 \% de la población de directivos, docentes de inglés y docentes de otras áreas, opinó que, si se identifican los indicadores motivación, autocontrol y habilidad social en esta dimensión, perteneciente a la variable Estrategias Didácticas de enseñanza del inglés. Así mismo, el 11,46\% de la población manifestó que no se identifican con estos indicadores, en la variable. Además, se puede resaltar que los indicadores 
Motivación y Habilidad Social representan la mayor fortaleza, desde la opinión de los directivos con un 93,93\% respectivamente, coincidiendo con la opinión de los docentes de otras áreas, con un $89,83 \%$ y con los docentes de inglés quienes le otorgan al indicador Motivación un 94,43\% respectivamente.

Igualmente se observa una moderada debilidad en el indicador Habilidad Social según la opinión de los docentes de otras áreas quienes le otorgan a este indicador un $79.67 \%$ y de acuerdo a lo expresado por los docentes de inglés un $83.33 \%$ coincidiendo ambos en el mismo indicador. Sin embargo, se nota que los docentes de otras áreas consideran dificultades al momento de identificarse con el autocontrol como factor relacionado al desempeño académico, otorgándole un $81,93 \%$.

En relación a los resultados obtenidos, los mismos coinciden con el antecedente de investigación de Cañizales (2011) el cual lleva por título "Diseño de material multimedia para el proceso de enseñanza aprendizaje del idioma inglés en educación media general”, y el cual arrojó como resultado que los directivos y docentes identifican, caracterizan, definen y describen las dimensiones de la variable. También se detectó que existen debilidades en algunos indicadores, revelando la necesidad imperante de elaborar un modelo de material multimedia para, de esta forma, fortalecer dichos indicadores y lograr un óptimo resultado en el proceso de enseñanza aprendizaje del inglés.

De tal manera que, este mismo resultado se puede evidenciar en la evaluación de esta dimensión, la cual arrojo también como resultado la necesidad imperante de elaborar un modelo o una propuesta que permita el mejoramiento de las habilidades sociales las cuales son un factor fundamental en el desempeño académico de los estudiantes.

Al comparar los resultados, en referencia a la debilidad que representa habilidad social, con los autores Peñafiel y Serrano (2005) quienes plantean que "las habilidades sociales son las conductas o destrezas sociales especificas requeridas para ejecutar competentemente una tarea de índole interpersonal y donde se pone en juego la interacción con otras personas (por ejemplo, hacer amigos)" (p. 10); los mismos difieren con sus tallados teóricos, pues se los docentes de inglés tienen cierta deficiencias en la promoción del desarrollo de destrezas sociales requeridas para interactuar con otras personas, brindar oportunidades para que sus estudiantes expresen sus sentimientos o deseos sobre su aprendizaje, y de igual manera enseñándoles diversas maneras correctas de comportamiento que variantes según cada persona.

Se observan diferencias con los resultados en referencia a la debilidad que representa el autocontrol, con el autor Reed y Kipp (2007) quienes plantea que "cómo ayudar a que los estudiantes se vuelvan más capaces de controlarse a sí mismos" (p.339); ya en la presente investigación los docentes de inglés, resaltan que existen fallas en prestar ayuda a los estudiantes en la orientación y conciencia de su autocontrol. 
Al analizar los datos obtenidos con el planteamiento del problema, se nota que hay coincidencias, debido a que existe una deficiencia en el desempeño académico de los estudiantes de inglés; y a raíz de los resultados, existe la necesidad de elaborar una propuesta que permita el mejoramiento de estos factores, determinantes en el desempeño académico de los estudiantes.

Dimensión: Tipos de estrategias didácticas.

Tabla 3. Tipos de estrategias didácticas.

\begin{tabular}{|c|c|c|c|c|c|c|}
\hline \multirow{2}{*}{$\begin{array}{l}\text { Alternativas } \\
\text { Población }\end{array}$} & \multicolumn{3}{|l|}{ SI } & \multicolumn{3}{|l|}{ NO } \\
\hline & Directivo & Docente & Docente Ingles & Directivo & Docente & Docente Ingles \\
\hline Indicadores & $\%$ & $\%$ & $\%$ & $\%$ & $\%$ & $\%$ \\
\hline Preinstruccionales & 90,9 & 90,37 & 94,43 & 9,1 & 9,63 & 5,57 \\
\hline Coinstruccionales & 90,9 & 85,87 & 94,43 & 9,1 & 14,13 & 5,57 \\
\hline Posinstruccionales & 81,8 & 74,6 & 55,53 & 18,2 & 25,4 & 44,47 \\
\hline Promedio & 87,87 & 83,61 & 81,47 & 12,13 & 16,39 & 18,53 \\
\hline Porcentaje & & & $84,31 \%$ & & & $15,69 \%$ \\
\hline
\end{tabular}

Fuente: Cuestionario

Elaborado por: Guerrero Keila, 2013

En la tabla anterior, se analiza la dimensión: tipos de estrategias de enseñanza, utilizadas actualmente por los profesores de inglés en Educación Media general para desarrollar el interés de los estudiantes por el aprendizaje de este idioma en el Municipio Escolar San francisco 1, del estado Zulia. Al respecto se observa que, el 84,31\% de la población de directivos, docentes de inglés y docentes de otras áreas opinaron, que se identifican estrategias didácticas tales como: las estrategias preinstruccionales, las coinstruccionales y las posinstruccionales. Así mismo, un $15,69 \%$ manifiesta no identificarse con estos indicadores en la variable.

En tal sentido, los resultados revelan que el indicador con mayor frecuencia relativa fue el referente a las estrategias preinstruccionales, con un 90,90\% de acuerdo a la opinión emitida por los directivos, 90,37\% por los docentes de otras áreas y 94,43\% por los docentes de inglés; mientras que el indicador Estrategias coinstruccionales, presentan frecuencia relativa de 90,90\% por los directivos, $85,87 \%$ por los docentes de otras áreas y $94,43 \%$ por los docentes de inglés.

Se presenta debilidad en el indicador Estrategias posinstruccionales con un $81,80 \%$ de acuerdo a la opinión emitida por los directivos, $74,60 \%$ por los docentes de otras áreas y 55,53\% por los docentes de inglés. Sin embargo, a pesar de que en su mayoría la población se identifica con los indicadores planteados; tanto los directivos, como los docentes de otras áreas y los docentes de inglés, consideran que hay diferencias marcadas y significativas en la puesta en práctica de las estrategias posinstruccionales en las clases de inglés. 
Al contrastar los resultados obtenidas de las dimensiones, se observa tener relación con lo planteado en la investigación de Luengo (2011) titulada: "Estrategias didácticas para la enseñanza del wayuunaiki en las instituciones educativas del Municipio Rosario de Perijá" en la que se concluye que la tendencia registrada por los docentes, utilizan las mismas estrategias en su gestión. Al identificar estas particularidades críticas, se sostiene que afectan de manera directa a la gestión de enseñanza, situación que impactaba de manera poco favorable al proceso de aprendizaje; propendiendo a diseñar una serie de acciones plenamente alineadas como una estrategia para la enseñanza del wayuunaiki.

De la misma forma, los resultados muestran que existe una necesidad de potenciar algunos tipos de estrategias como las posintruccionales, las cuales se revelarán en el marco de la propuesta de estrategias didácticas de enseñanza del inglés, para las instituciones del Municipio San Francisco 1 , a través de la ejecución de acciones diseñadas en el mismo.

Al comparar los resultados en referencia al indicador que obtuvo mayor debilidad, con los postulados teóricos, se puede resaltar que, González (2006) plantea que las estrategias posinstruccionales "se utilizan después de una enseñanza de un material de aprendizaje, permite que el alumno se forme una visión sintética y global de la información" (p.18). Por ello, es sumamente necesario la utilización de las estrategias para poder consolidar los aprendizajes en los estudiantes, fomentando la formación de una visión o idea global de lo estudiado y así pueda fijarse de manera permanente en ellos.

En tal sentido, los resultados no coinciden con los postulados teóricos, donde refleja que los docentes de inglés, en su mayoría tienen deficiencias al desarrollar la reflexión al final de la clase, por parte de los estudiantes, y el desarrollo de una visión global de la información. Lo cual justifica el diseño de una propuesta que ayude al mejoramiento y puesta en práctica de estas estrategias de enseñanza que serán vitales en el proceso de formación de los estudiantes de inglés.

Al contrastar estos resultados con el planteamiento del problema, se nota que hay coincidencia, debido a que existe una deficiencia en la práctica docente con respecto al uso de las estrategias didácticas de enseñanza; y sobre la base es estos, se concluye que si hay la necesidad de elaborar una propuesta que permita el mejoramiento de estas estrategias las cuales son determinantes en el proceso de enseñanza-aprendizaje del inglés. 


\section{Dimensión: Competencia Comunicativa}

Tabla 4. Competencia Comunicativa

\begin{tabular}{|c|c|c|c|c|c|c|}
\hline Alternativas & SI & & & NO & & \\
\hline Población & Directivo & Docente & $\begin{array}{l}\text { Docente } \\
\text { Ingles }\end{array}$ & Directivo & Docente & $\begin{array}{l}\text { Docente } \\
\text { Ingles }\end{array}$ \\
\hline Indicadores & $\%$ & $\%$ & $\%$ & $\%$ & $\%$ & \\
\hline Expresión Oral & 90,9 & 86,97 & 77,77 & 9,1 & 13,03 & 22,23 \\
\hline Expresión Escrita & 96,97 & 85,33 & 66,67 & 3,03 & 14,67 & 33,33 \\
\hline Comprensión Oral & 93,93 & 82,5 & 88,9 & 6,07 & 17,5 & 11,1 \\
\hline Comprensión Escrita & 90,9 & 89,23 & 55,57 & 9,1 & 10,77 & 44,43 \\
\hline Promedio & 93,18 & 86,01 & 72,23 & 6,83 & 13,99 & 27,78 \\
\hline Porcentaje & & & $83,80 \%$ & & & $16,20 \%$ \\
\hline
\end{tabular}

Fuente: Cuestionario

Elaborado por: Guerrero Keila, 2013

En la tabla, analizando la dimensión con respecto a definir las competencias comunicativas desarrolladas en el aprendizaje de una segunda lengua por los profesores de inglés en Educación Media General del Municipio Escolar San Francisco 1 del estado Zulia"; se registra que el 83,80\% de la población de directivos, docentes de inglés y docentes de otras áreas opinaron que, se identifican con los indicadores, expresión oral, expresión escrita, comprensión oral y comprensión escrita; mientras que el 16,20\% de la población manifestó no identificarse.

En consonancia con lo anterior, se resalta que el indicador expresión escrita, con el 96,97\% de frecuencia de respuestas, presentan la mayor fortaleza según la opinión emitida por los directivos; mientras que, con los docentes de otras áreas, el indicador con más fortaleza corresponde a la comprensión escrita con el 89,23\%. Finalmente, se encuentra el indicador comprensión oral con un $88,90 \%$.

Se presenta una ligera debilidad o cierta dificultad en el desarrollo del indicador, comprensión oral, según la opinión de los docentes de otras áreas, colocándolo en un $82.50 \%$. Así mismo, el indicador Comprensión escrita, según lo expresado por los docentes de inglés, presenta una gran debilidad o dificultad en su práctica, al tener el menor porcentaje, un 55,57\%, la expresión escrita con un $66,67 \%$ y la expresión oral con el $77,77 \%$ de frecuencia de respuestas. Lo que requiere un análisis exhaustivo sobre cómo lograr transformar las dificultades o debilidades, en fortalezas que permitan que el proceso de enseñanza-aprendizaje del inglés, se mejore y pueda estar en su más alto nivel de calidad; para ello se hace pertinente la elaboración de una propuesta que permita lograr tales objetivos.

En relación con lo expuesto en la investigación realizada por Ferrer (2008) la cual llevó por título "Estrategias de comprensión lectora para estudiantes de Educación Media, Diversificada y Profesional en el área de Ingles" Obteniendo resultados que evidenciaron limitaciones para alcanzar la comprensión lectora de textos en inglés y la poca utilización de estrategias cognitivas 
y metacognitivas por parte de los docentes. De tal manera que el estudio coincide con la dimensión comprensión escrita la cual alcanzó mayor debilidad. Por ello, que se hace pertinente la construcción de una propuesta que permita, no mejorar las competencias comunicativas, no solo de la comprensión escrita sino también de la expresión oral y escrita; para así obtener mejor desempeño comunicativo del inglés, en Educación Media General.

Al contrastar los resultados en referencia a la debilidad que representa la comprensión escrita, con el autor Pérez (2007) quien establece que la comprensión escrita "comprende la habilidad que permite, a partir de distintos tipos de textos, buscar, recopilar y procesar información y ser competente a la hora de comprender" (p.65), se determina que, los resultados difieren con los postulados teóricos, ya que se observa un gran porcentaje de dificultad en la práctica de la comprensión escrita, por parte de los docentes de inglés.

Se presume que los docentes, generan pocas expectativas sobre el contenido de un texto, muy poco introducen en tópicos interesantes que motiven la lectura y promuevan el dialogo entre autores y lectores, a través de la experiencia de la lectura. Por otra parte, el desarrollo de la expresión oral, según lo planteado por Harmer (2007):

Proporciona oportunidades para ensayar y practicar conversaciones de la vida real, dentro de un ambiente seguro como lo es el aula de clases. Así como también, promueve "feedback" (retroalimentación) entre el profesor y los estudiantes y es un espejo para visualizar los aciertos y errores que se están cometiendo y también permite activar varios elementos del lenguaje en el cerebro para convertir al estudiante en un usuario del lenguaje, más autónomo (p.123).

De esta manera, al confrontar los resultados obtenidos, se observa que no existe concordancia, ya que no se está logrando lo que éste afirma con los estudiantes en el desarrollo de la dimensión, ya que hay ciertas dificultades por parte de los docentes, para crear actividades que promuevan el procesamiento o interpretación de lo escuchado, así como también para practicar conversaciones de la vida real en el idioma inglés a través del intercambio del dialogo y emisión de enunciados.

Otra debilidad observada, fue la dimensión Expresión escrita, la cual confrontándola con el autor Ardila y Rosselli (2005) estos afirman que:

La escritura no se limita solo a un movimiento efectuado por la mano, por complejo que este sea, ni al dominio del sistema ortográfico de la lengua; la escritura implica acciones organizadas y precisas que permiten representar de manera simultánea las características fonológicas, semánticas, sintácticas y pragmáticas del lenguaje oral. (p.25)

Por lo que, contrastando con los resultados obtenidos, se observa que hay divergencia en los postulados con la práctica de los mismos, ya que se puede deducir que el docente, poco impulsa en sus estudiantes la expresión de sus pensamientos e ideas, plasmándolos en un papel; así como también tiene dificultad para utilizar la escritura como herramienta práctica para el procesamiento del lenguaje, por parte de los estudiantes. 
En este mismo sentido, y al confrontar los resultados con el planteamiento del problema, se nota que hay coincidencia, debido a que existe una dificultad por parte de los docentes de inglés, en el logro de los indicadores que denotan la competencia comunicativa en los estudiantes; y a raíz de estos resultados, se deduce que si hay la necesidad de elaborar una propuesta que permita el mejoramiento de estos indicadores fundamentales y determinantes en el aprendizaje del idioma.

Variable: Estrategias Didácticas para la enseñanza del inglés en Educación Media General.

Tabla 5. Estrategias Didácticas para la enseñanza del inglés en Educación Media General.

\begin{tabular}{|c|c|c|c|c|c|c|}
\hline Alternativas & SI & & & NO & & \\
\hline Población & Directivo & Docente & $\begin{array}{l}\text { Docente } \\
\text { Ingles }\end{array}$ & Directivo & Docente & $\begin{array}{l}\text { Docente } \\
\text { Ingles }\end{array}$ \\
\hline Dimensiones & $\%$ & $\%$ & $\%$ & $\%$ & $\%$ & $\%$ \\
\hline $\begin{array}{l}\text { Factores relacionados al } \\
\text { D.A }\end{array}$ & 92,92 & 83,81 & 88,88 & 7,08 & 16,19 & 11,12 \\
\hline Tipos de Estrategias & 87,87 & 83,61 & 81,47 & 12,13 & 16,39 & 18,53 \\
\hline Competencia Comunicativa & 93,18 & 86,01 & 72,23 & 6,83 & 13,99 & 27,78 \\
\hline Promedio & 91,33 & 84,47 & 80,86 & 8,68 & 15,52 & 19,14 \\
\hline Porcentaje & & & $85,56 \%$ & & & $14,44 \%$ \\
\hline
\end{tabular}

Fuente: Cuestionario

Elaborado por: Guerrero Keila, 2013

En el análisis de la variable, Estrategia didácticas de enseñanza del inglés en Educación Media General, se observa que el $85,56 \%$ de los encuestados manifestaron que se sienten identificados. Sin embargo, existe cierta debilidad, donde se observa que el $14,44 \%$ no se identifica con dichas estrategias didácticas.

La dimensión con mayor frecuencia relativa son los factores que determinan el desempeño académico con el 92,92\% según la opinión establecida por los directivos y el 88,88\% según lo que opinan los docentes de inglés. Por otra parte, la dimensión la que refleja mayor debilidad, según la opinión de los docentes de inglés con el 72,23\% es la referente a la competencia comunicativa.

Así mismo, el indicador (tipos de estrategias) presenta una ligera debilidad en relación a la opinión emitida por los docentes de otras áreas, quienes le otorgaron a esta el 83,61\% y los docentes de inglés quienes opinaron con el $81,47 \%$ de frecuencia de respuestas. Lo que permite determinar que la dimensión que necesita ser potenciada, corresponde a la Competencia Comunicativa. En este sentido, Orozco (2010), plantea que la competencia comunicativa:

Abarca tanto el conocimiento de la lengua como la habilidad para utilizarla. Comprende la experiencia social, las necesidades y motivaciones y el acceso y selección de la información; y se resume a la capacidad de hacer bien el proceso de Comunicación (p.2). 
De tal manera que según lo expresado por el autor y los resultados obtenidos, hay una gran debilidad en el desarrollo de la competencia comunicativa en los estudiantes de Educación Media general, ya que existen cierto desconocimiento de la lengua y el desarrollo de la habilidad para usarla, permitiendo así un buena comunicación en el idioma, por parte de los estudiantes; y esto es muy grave debido a que la competencia comunicativa es una de las dimensiones más determinantes en el logro de la comunicación eficaz en cualquier idioma.

En consonancia con lo anterior, y haciendo un contraste de los resultados con el planteamiento del problema, se nota que si hay concordancia ya que en éste se planteaba que los estudiantes no estaban adquiriendo las habilidades de comunicación en su mayor nivel, lo cual indicaba que podrían existir ciertas deficiencias en la práctica de la comunicación en las aulas, en el idioma extranjero.

\section{Conclusiones}

Se pudo determinar que los directivos, docentes de inglés y docentes de otras áreas, si se identifican los indicadores motivación, autocontrol y habilidad social como factores determinantes en el desempeño académico. Así mismo, el $11.46 \%$ de la población manifestó que no se identificó con estos indicadores, en la variable. Además, se pudo notar que tanto la Motivación como la Habilidad Social representan la mayor fortaleza, según la opinión de los directivos y los docentes de otras áreas, quienes coincidieron con los docentes de inglés en el indicador Motivación. Igualmente se observó una moderada debilidad en el indicador Habilidad Social según la opinión de los docentes de otras áreas y los docentes de inglés. Sin embargo, se nota que los docentes de otras áreas consideran dificultades al momento de identificarse con el autocontrol como factor relacionado al desempeño académico.

La población opinó que, si se identifican con los tipos de estrategias didácticas de enseñanza, dentro de las cuales están: las estrategias preinstruccionales, las coinstruccionales y las posinstruccionales. Además, el 15.69\% manifestó no identificarse con estos indicadores en la variable. El indicador con mayor frecuencia relativa fue el referente a las estrategias preinstruccionales; mientras que se presentó debilidades en el indicador Estrategias posinstruccionales de acuerdo a la opinión emitida por los directivos, por los docentes de otras áreas y por los docentes de inglés. Lo cual indica que a pesar de que, en su mayoría, la población se identificó con los indicadores planteados; hubo diferencias visibles y significativas en la puesta en práctica de las estrategias posinstruccionales en las clases de inglés, lo cual promovió el diseño de una propuesta que permitiera neutralizar esta falla.

Con respecto al desarrollo de las competencias comunicativas en el aprendizaje de una segunda lengua por los profesores de inglés en Educación Media General, se concluyó que hay debilidad o dificultad principalmente en el desarrollo de la Comprensión escrita, seguidamente se encontró cierta debilidad en la expresión escrita y la expresión oral, según lo expresado por los docentes de inglés. Posteriormente, al hacer el análisis, se observó que la dimensión con mayor frecuencia 
relativa fueron los factores que determinan el desempeño académico, según la opinión emitida por los directivos y los docentes de inglés; mientras la dimensión que presenta mayor debilidad y que necesita ser potenciada fue las Competencia Comunicativas.

\section{Referencias bibliográficas}

Alcedo, Y. (2002). Enseñanza del idioma inglés en niños de la Primera Etapa de Educación Básica. Ponencia presentada en el XVIII Encuentro Nacional de Educadores. Mérida. Venezuela

Alfonzo, A. (2003). Estrategias instruccionales. (s/e)

Ardila, A., Rosselli, M. (2005). Neuropsicología de los trastornos del aprendizaje.

Baralo, M. (2000). La adquisición del español como lengua extranjera. Madrid. ArcoLibro.

Barragán, R., Salman, T., Ayllon, V., Córdova, J., Langer E., Sanjinés, J., Rojas, R. (2003). Guía para la formulación y ejecución de proyectos de investigación. Editorial Offset Boliviana Ltda. (3ra edición)

Boix, R. (1999). Estrategias y recursos didácticos en la escuela rural. Editorial GRAO.

Carbonel, R. (2008). Leer escribir y hablar. Editorial Edaf S.L

Chacón, C. (2003). Hacia el desarrollo de una práctica pedagógica reflexiva mediante la actualización de los docentes de inglés de la 3ra etapa de educación básica. Evaluación de una experiencia. Acción pedagógica

Gómez, J. (2005). La popularidad del inglés en el siglo XXI. Universidad Autónoma de San Luis Potosí.

Guerra R. (2004). Experiencias didácticas en el aprendizaje del inglés en la 2da etapa de educación básica.

Harmer J. (2007). How to teach English. Logman

Hernández, S., Fernández C., Baptista M. (2006). Metodología de la investigación. México. McHill Interamericana.

Orellana, A. (2008). Estrategias en educación. Venezuela. Editorial Mc Graw Hill.

Orozco, L. (2010) Articulo: Importancia de las competencias: Lingüística, comunicativa y pragmática.

Peñafiel, E., Serrano, C. (2005). Habilidades Sociales. Editorial Editex.

Pérez, P. (2007). La competencia de las competencias: la comunicación lingüística, su presencia en el currículo de la educación infantil y primaria. Editorial Edigrafos. 
Quispe, A. (2004). Evaluación socioeconómica de programas de desarrollo / Socio-Económica. Editorial PYV

Reed, D. Kipp, K. (2007). Psicología del desarrollo. Infancia y adolescencia (7ma Edición).

Zamora, R. (2009) Como enseñar castellano. Edit Universidad estatal a distancia, Costa Rica.

González, F. (2008). Comunicación y Educación en la enseñanza del inglés. Universidad Pedagógica Nacional. Hermosillo. México

Ferrer, G. (2008). Estrategias de comprensión lectora para estudiantes de Educación Media, Diversificada y Profesional en el área de inglés. La Universidad del Zulia.

Cañizales, O. (2011). Diseño de material multimedia para el proceso de enseñanza aprendizaje del idioma inglés en educación media general. Universidad Rafael Urdaneta. Venezuela.

Luengo, E. (2011). Estrategias didácticas para la enseñanza del Wayuunaiki en las instituciones educativas. La Universidad del Zulia. Venezuela.

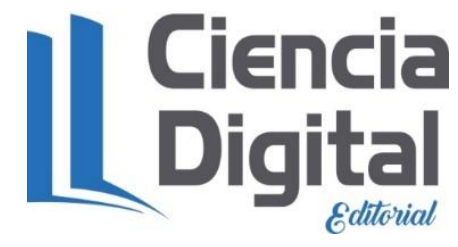




\section{PARA CITAR EL ARTÍCULO INDEXADO.}

Guerrero Chacón, K., Vallejo Barreno, C., \& Caicedo Valencia, M. (2019). Estrategias didácticas para la enseñanza del inglés en educación media general. Ciencia Digital, 3(4.2), 25 - 42. https://doi.org/10.33262/cienciadigital.v3i4.2.1001

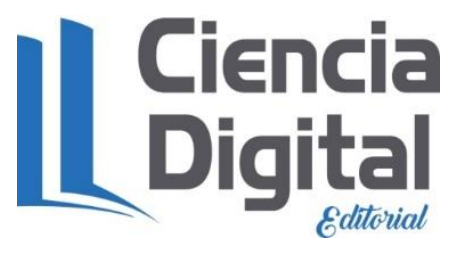

El artículo que se publica es de exclusiva responsabilidad de los autores y no necesariamente reflejan el pensamiento de la Revista Ciencia Digital.

El artículo queda en propiedad de la revista y, por tanto, su publicación parcial y/o total en otro medio tiene que ser autorizado por el director de la Revista Ciencia Digital.
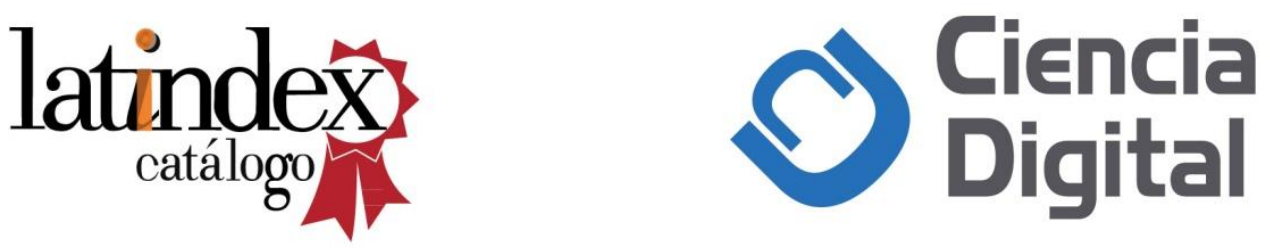